\title{
Article
}

Mycosphere

Doi 10.5943/mycosphere/7/2/10

Copyright (C) Guizhou Academy of Agricultural Sciences

\section{The phenotypic and metabolic properties of Metarhizium guizhouense on Corcyra cephalonica}

\section{Thaochan $\mathbf{N}^{1}$ and Chandrapatya $\mathrm{A}^{2}$}

${ }^{1}$ Department of Pest Management, Faculty of Natural Resources, Prince of Songkla University, Hat Yai campus, Songkhla, 90112, Thailand.

${ }^{2}$ Department of Entomology, Faculty of Agriculture, Kasetsart University, Jatuchak, Bangkok, 10900, Thailand.

Thaochan N, Chandrapatya A 2016 - The phenotypic and metabolic properties of Metarhizium guizhouense on Corcyra cephalonica. Mycosphere 7(2), 214-225, Doi 10.5943/mycosphere/7/2/10

\begin{abstract}
An entomopathogenic fungus, Metarhizium guizhouense, used as biological control agent for insect pests. The phenotypic (mycelial growth and sporulation) and metabolic properties (enzyme production and virulence) of $M$. guizhouense PSUM02 and PSUM04 were compared after successive subcultures on an artificial medium (5 or 10 cycles), and after passages through larvae of an insect host, Corcyra cephalonica (3, 5 or 7 cycles). The mycelial growth rates were not significantly affected, with the exceptions of PSUM02 subcultured 10 times and PSUM04 subcultured 5 times, both of which had slightly reduced mycelial growth rates. Passages through an insect host gave the highest spore production, similar to the original fungal culture, while subculturing on an artificial medium reduced spore production several fold. Only after 5 and 7 passages through an insect host did PSUM02 have chitinase activity, while with other treatments (including no treatment of the original fungal culture) it had no chitinase activity. Successive subculturing of $M$. guizhouense PSUM02 and PSUM04 on an artificial medium for 10 cycles decreased the virulence of the fungus compared to the original culture, while passages through an insect hosts increased the virulence. The results indicate that the virulence of M. guizhouense PSUM02 and PSUM04 passages through an insect host for 3 times suitable for restoring the phenotypic and metabolic properties for biological control agent in insect pest control.
\end{abstract}

Key words - enzyme production - passage through insect - repeated subcultures - virulence

\section{Introduction}

Metarhizium guizhouense Q.T. Chen \& H.L. Guo is an entomopathogenic fungus belonging to the M. anisopliae complex (Bischoff et al. 2009). This fungal species is closely related to currently recognized varieties of $M$. anisopliae as well as to $M$. pingshaense and to $M$. taii (Bischoff et al. 2009). Some species of Metarhizium are commonly used to control various insect pests of plants and livestock (Boucias \& Pendland 1998), and they have potential for use against hemipteran (Geng \& Zhang 2004), lepidopteran (Loc \& Chi 2007, Balachander et al. 2012, Petlamul \& Prasertsan 2012), coleopteran (Lui \& Bauer 2006, Khashaveh et al. 2011), dipteran insects (Jonason et al. 2005, Yousef et al. 2013, Thaochan \& Ngampongsai 2015), and mite pests (Wekesa et al. 2005, Tavassoli et al. 2011).

One major problem in using entomopathogenic fungi is their variation in pathogenesis and virulence. Some strains lose their virulence after subculturing and passages through artificial media, 
and such cultivation in artificial media causes phenotypic alterations associated with degeneration of the cultures (Song \& Feng 2011). The phenotypic alterations include changes in spore color, sporulation, mycelial growth, and colony morphology. Fungal properties such as sporulation, enzyme activity and virulence are important in the use of entomopathogenic fungi; however, these can degenerate with culturing on an artificial medium (Butt et al. 2006).

Successive subculturing on an artificial medium is known to reduce the virulence of entomopathogenic fungi (Frazzon et al. 2000, Shah et al. 2005, Butt et al. 2006). Some strains lose their virulence after one or two cycles of subculturing while others can be subcultured more than 10 cycles before their virulence declines (Brownbridge et al. 2001, Vandenberg \& Cantone 2004). Re-inoculation of the fungus in a suitable insect host may improve or restore the virulence properties (Brownbridge et al. 2001, Vandenberg \& Cantone 2004, Adames et al. 2011, Song \& Feng 2011).

Rice moth, Corcyra cephalonica Stainton (Lepidoptera; Pyralidae), is an important insect pest of stored rice, bean, corn and some grains (Jagadish et al. 2009), and the eggs of this insect are used in laboratories to feed its natural enemies, such as Trichogramma sp. and lacewing (Parra et al. 1991).

The aim of this research was to assess the effects of subculturing in an artificial media, and passaging through rice moth larvae, C. cephalonica, on the key pest control characteristics of the $M$. guizhouense fungus. The observed characteristics were mycelial growth, sporulation, enzyme production and virulence.

\section{Materials and Methods}

\section{Fungal strains and conidia preparation}

Two Metarhizium guizhouense isolates, PSUM02 and PSUM04, were obtained from the National Biological Control Research Center, Southern Region, Faculty of Natural Resources, Prince of Songkla University, Hat Yai, Thailand. These had been isolated from insect cadavers of Tibicen sp. (Hemiptera: Cicadidae) and Brontispa longissima Gestro (Coleoptera: Chrysomelidae). The fungal isolates were confirmed by PCR amplification and sequencing of the elongation factor1 alpha (EF-1 $\alpha)$ gene (Bidochka et al. 2001, Bidochka et al. 2005), and comparison to the Metarhizium species in the GenBank database (accession numbers AB981657 and AB981658 are now assigned to PSUM02 and PSUM04). The stock culture was maintained on Sabouraud dextrose agar plus $2 \%$ of yeast extract (SDAY) at $4{ }^{\circ} \mathrm{C}$ until use.

\section{Repeated subculturing on artificial medium}

M. guizhouense PSUM02 and PSUM04 were grown on SDAY at $28.0 \pm 2.0^{\circ} \mathrm{C}$ until sporulation (14 days). The conidia were harvested from the SDAY plates by scarping with a loop, and sub-cultured to fresh SDAY plates. This multi-spore in vitro transfer was repeated for 5 and 10 sub-cultures. The fungi from each of these two treatments, labeled as "Sub5" and "Sub10" were kept on similar medium in an incubator at $28.0 \pm 2.0^{\circ} \mathrm{C}$ until use in experiments.

\section{Passaging through an insect host}

Conidia suspensions $\left(1 \times 10^{8}\right.$ conidia/ml $)$ were prepared from the original stock cultures of $M$. guizhouense PSUM02 and PSUM04. Six instar larvae of $C$. cephalonica were dipped in the conidia suspension for one min, and allowed to dry on sterile paper towels, then placed in clean Petri dishes with sterile filter paper $\left(\right.$ Whatman $^{\circledR} \# 1$ ) and incubated at $28.0 \pm 2.0^{\circ} \mathrm{C}, 70-90 \% \mathrm{RH}$ and $12: 12$ (light : dark). The conidia from cadavers were harvested by scraping with a loop, suspended in $0.1 \%(\mathrm{w} / \mathrm{v})$ Tween 80 , and used to inoculate $\left(1 \times 10^{8}\right.$ conidia/ml $)$ additional insects. This insect passage was repeated 3, 5 and 7 times. The fungi from each treatment, labeled as "In3", "In5" and "In7”, were kept on similar medium in an incubator at $28.0 \pm 2.0^{\circ} \mathrm{C}$ until use in experiments. 

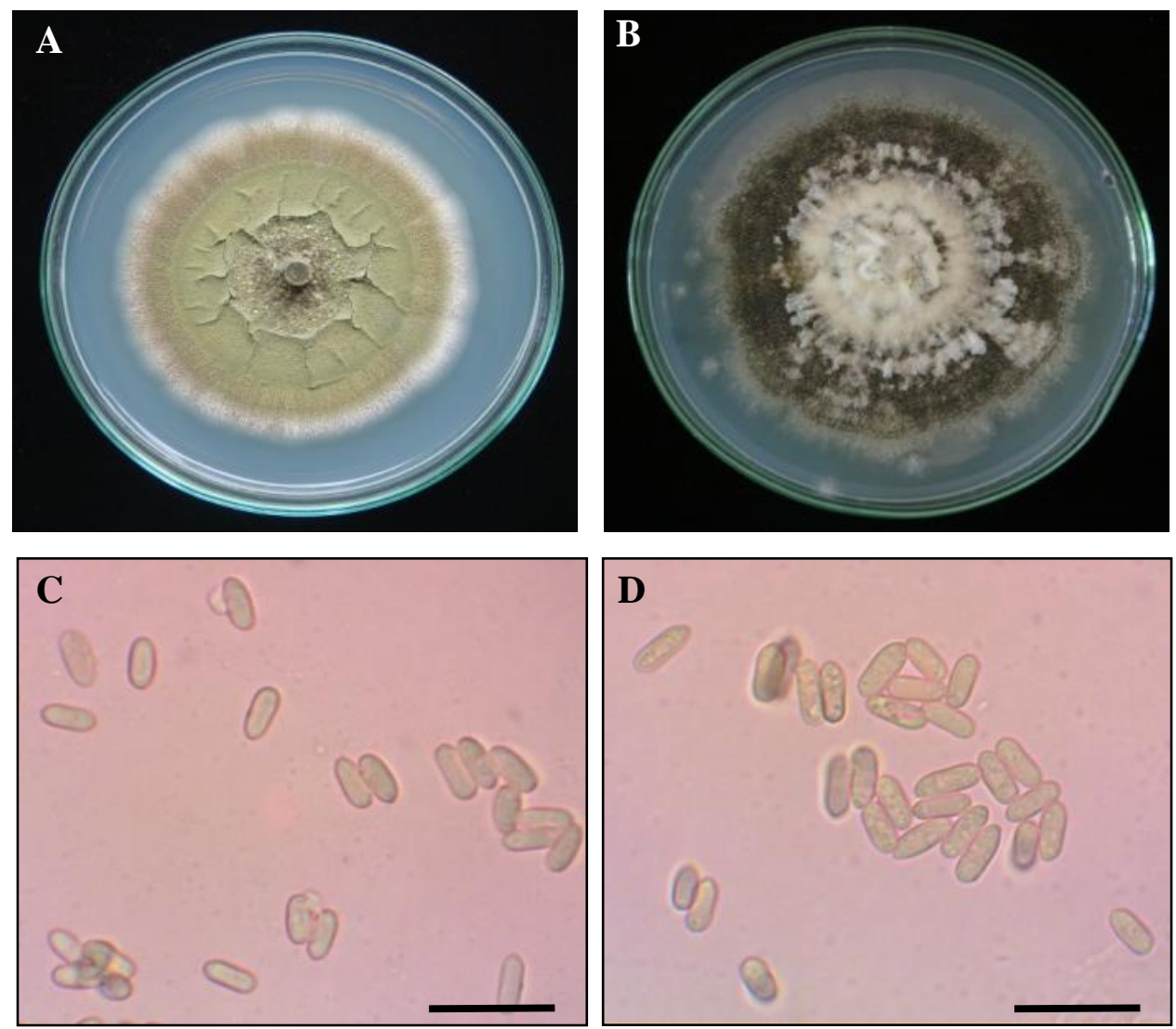

Fig. 1 - Colonial and conidial morphology of initial stock of Metarhizium guizhouense PSUM02 (A and C) and PSUM04 (B and D) on SDAY medium at 20 days. M. guizhouense PSUM02 is flat colony, smooth mycelial and conidial mass like a mat with a light green color. M. guizhouense PSUM04 is flat colony, smooth mycelial and zonation of conidia mass with dark green color. Scale bar $=10 \mu \mathrm{m}$.

\section{Mycelial growth and spore production}

The effects of the treatments on M. guizhouense PSUM02 and PSUM04 mycelial growth and spore production were assessed by comparison with the original stock. Spore suspensions $(0.1$ $\mathrm{ml}$ of $1 \times 10^{8}$ spore/ml) were spread on SDAY plates, and incubated at previously described conditions for three days to obtain mycelial mats. Unsporulated mycelial mat samples were cut from the culture plates using a $7 \mathrm{~mm}$ diameter cork borer. Each agar plug was then transferred onto the center of a fresh $90 \mathrm{~mm}$ diameter SDAY agar plate, and incubated similarly as before. Radial growth (in $\mathrm{mm}$ ) was recorded at five day intervals for up to 20 days, by measuring two cardinal diameters along orthogonal axes pre-drawn on the bottom of each plate.

To assess spore production, treated or original stock was washed with $100 \mathrm{ml}$ sterile distilled water (with $0.01 \%(w / v)$ Tween 80 ) and spread on a SDAY plate. The spore density in suspension was counted at 5,10,15 and 20 days, using a hemocytometer under a compound microscope.

\section{Chitinase production assay}

\section{Colloidal chitin preparation}

Ten grams of commercial chitin from shrimp shell powder (Sigma, No. C7170) was added to $100 \mathrm{ml}$ of $85 \%$ phosphoric acid, and held at $4{ }^{\circ} \mathrm{C}$ for $24 \mathrm{~h}$. Thereafter, two liters of tap water was added. The liquid was separated from the gelatinous white material by filtration through filter paper, and the filter cake was washed with tap water until the filtrate had a pH of 6.5. The colloidal chitin obtained had a soft, pasty consistency and 90-95\% moisture. 
Table 1 The mycelial growth rates (mm/day) of M. guizhouense PSUM02 and PSUM04 repeatedly passaged through $C$. cephalonica larvae or serially subcultured on artificial media after inoculation on SDAY media. Refer to the Materials and Methods section for details of the M. guizhouense PSUM02 and PSUM04 treatments.

\begin{tabular}{lllllll}
\hline \multirow{2}{*}{ Fungal isolate } & Treatment & \multicolumn{2}{l}{ Radius growth rate $(\mathrm{mm} / \mathrm{day})^{2}$} & & \\
\cline { 3 - 6 } & & Day 5 & Day 10 & Day 15 & Day 20 & Slope $( \pm$ SD $)$ \\
\hline \multirow{2}{*}{ PSUM02 } & Stock & $4.9 \pm 0.1 \mathrm{a}$ & $4.5 \pm 0.1 \mathrm{a}$ & $4.6 \pm 0.1 \mathrm{a}$ & $3.9 \pm 0.0 \mathrm{a}$ & $-0.31 \pm 0.04 \mathrm{bcd}$ \\
& Sub5 & $4.5 \pm 0.1 \mathrm{~d}$ & $4.0 \pm 0.0 \mathrm{~b}$ & $3.9 \pm 0.1 \mathrm{c}$ & $3.5 \pm 0.1 \mathrm{~b}$ & $-0.31 \pm 0.05 \mathrm{bcd}$ \\
& Sub10 & $4.6 \pm 0.1 \mathrm{~cd}$ & $4.1 \pm 0.1 \mathrm{~b}$ & $3.9 \pm 0.2 \mathrm{c}$ & $3.5 \pm 0.1 \mathrm{~b}$ & $-0.34 \pm 0.06 \mathrm{abcd}$ \\
& In3 & $4.9 \pm 0.2 \mathrm{ab}$ & $4.6 \pm 0.1 \mathrm{a}$ & $4.5 \pm 0.0 \mathrm{a}$ & $4.0 \pm 0.0 \mathrm{a}$ & $-0.28 \pm 0.05 \mathrm{~cd}$ \\
& In5 & $4.9 \pm 0.1 \mathrm{ab}$ & $4.5 \pm 0.1 \mathrm{a}$ & $4.5 \pm 0.0 \mathrm{a}$ & $3.9 \pm 0.1 \mathrm{a}$ & $-0.29 \pm 0.03 \mathrm{~cd}$ \\
& In7 & $4.6 \pm 0.1 \mathrm{~cd}$ & $4.1 \pm 0.1 \mathrm{~b}$ & $4.1 \pm 0.1 \mathrm{~b}$ & $3.8 \pm 0.1 \mathrm{a}$ & $-0.26 \pm 0.04 \mathrm{~d}$ \\
\hline \multirow{3}{*}{ PSUM04 } & Stock & $4.6 \pm 0.1 \mathrm{bcd}$ & $3.9 \pm 0.1 \mathrm{~b}$ & $3.6 \pm 0.1 \mathrm{~d}$ & $3.5 \pm 0.0 \mathrm{~b}$ & $-0.39 \pm 0.04 \mathrm{ab}$ \\
& Sub5 & $4.7 \pm 0.1 \mathrm{abcd}$ & $4.0 \pm 0.0 \mathrm{~b}$ & $3.8 \pm 0.1 \mathrm{~cd}$ & $3.5 \pm 0.1 \mathrm{~b}$ & $-0.37 \pm 0.05 \mathrm{abc}$ \\
& Sub10 & $4.7 \pm 0.1 \mathrm{abcd}$ & $4.0 \pm 0.1 \mathrm{~b}$ & $3.8 \pm 0.1 \mathrm{~cd}$ & $3.5 \pm 0.1 \mathrm{~b}$ & $-0.40 \pm 0.05 \mathrm{ab}$ \\
& In3 & $4.7 \pm 0.1 \mathrm{abcd}$ & $4.1 \pm 0.1 \mathrm{~b}$ & $3.8 \pm 0.1 \mathrm{c}$ & $3.4 \pm 0.1 \mathrm{~b}$ & $-0.39 \pm 0.02 \mathrm{ab}$
\end{tabular}

${ }^{1}$ Sub5 = subculture on SDAY plates 5 times; Sub10 = subculture on SDAY plates 10 times; In3 = infected through an insect 3 times; In5 = infected through an insect 5 times; and In $7=$ infected through an insect 7 times. Stock = initial stock culture.

${ }^{2}$ Numbers are mean \pm SD. Different letters within a column indicate statistically significant differences at $P<0.01$, according to Tukey's HSD test.

\section{Fungal preparation and chitinase production assay}

For in-vitro chitinolytic enzyme plate assays, treated and control spore suspensions $(0.1 \mathrm{ml}$ of $1 \times 10^{6}$ spore/ml) were spread on SDAY plates and incubated in dark for three days at $28.0 \pm$ $2.0^{\circ} \mathrm{C}$ to obtain mycelial mats. Four unsporulated mycelial mat samples were then cut from the culture plates using a seven mm diameter cork borer. Each agar plug was then transferred onto the center of a fresh $90 \mathrm{~mm}$ diameter plate of SDAY plus $2 \%(\mathrm{w} / \mathrm{v})$ colloidal chitin, and incubated at the conditions described above. Enzyme production was determined after five days by staining the gel with $0.1 \%$ (w/v) of Congo Red solution for 30 min followed by washing with $1 \mathrm{~N} \mathrm{NaCl}$. The enzyme production was assigned an enzymatic index by dividing the total diameter (in $\mathrm{mm}$ ) of the colony plus halo by the diameter of the colony. The presence of a clear zone, a halo around the colony, was an indicator of chitinase production, with a larger halo corresponding to higher activity, and an enzymatic index value $>1.0$ indicated activity (St. Leger et al. 1997).

\section{Protease production assay}

Three-day-old unsporulated mycelial mats from each treatment were each transferred onto the center of a fresh SDAY plus $3 \%(\mathrm{w} / \mathrm{v})$ skim milk powder, and incubated similarly as in the chitinase assay. The presence of a clear zone (in $\mathrm{mm}$ ) was an indicator of protease production, and the enzymatic index was based on observations at five days of incubation, the calculations following St. Leger et al. (1997).

\section{M. guizhouense virulence on C. cephalonica larvae assays}

Ten larvae were used per treatment, and their live/dead statuses were observed over time after exposure. Spore suspensions of treated and original stock cultures were set up with $1 \times 10^{8}$ spore $/ \mathrm{ml}$ in sterile distilled water with $0.01 \%(\mathrm{w} / \mathrm{v})$ Tween 80 . Ten $6^{\text {th }}$ instar $C$. cephalonica larvae were dipped into each spore suspension for one min, transferred onto sterile towel paper, and when completely dry they were placed in clean clear plastic boxes $(10 \times 10 \times 10 \mathrm{~cm})$ with a hole on the lid 
for air ventilation. The rearing conditions were $28.0 \pm 2.0^{\circ} \mathrm{C}, 70-90 \% \mathrm{RH}$, and $12: 12$ (light : dark). The live/dead statuses of the larvae were observed and recorded daily. Each dead larva was placed on moist filter paper (Whatmann ${ }^{\circledR} \# 1$ ) in a sterile Petri dish, where the emergence of fungal hyphae and conidia were observed. For each treatment, the mortality experiment with ten larvae was replicated ten times.

\section{Statistical analyses}

A total of 12 treatments were compared by analysis of variance (ANOVA) for their effects on mycelia growth, sporulation, chitinase and protease productions, and the virulence on $C$. cephalonica larvae. For each of the fungi, $M$. guizhouense PSUM02 and PSUM04, the six treatments corresponded to the following: control "Stock"; samples repeatedly passaged through $C$. cephalonica larvae, "In3", "In5", and "In7"; and samples that had been repeatedly subcultured, "Sub5" and "Sub10".

The assays for mycelia growth, sporulation, and the two enzyme productions each had four replicates, and the virulence of the fungi on $C$. cephalonica larvae was replicated ten times (each replicate using ten larvae per treatment group). For each fungal treatment, the average survival time (AST) with 95\% confidence limits of infected $C$. cephalonica larval population was calculated by Kaplan-Meier survival analysis from the mortality percentages recorded each day after inoculation. These results were compared using ANOVA, and the mean results of each treatment group were compared using the Tukey's Honestly Significant Difference test $(\alpha=0.05)$. Regression analysis was used for correlation assessments. The analyses were carried out using the SPSS 11.0 program for Windows (SPSS, 2001).

\section{Results}

\section{Colony morphology}

The two strains of M. guizhouense, PSUM02 and PSUM04, differed in their mycelia and sporulation patterns on culture media (Fig. 1). Treated PSUM02 colonies were flat and the conidia were mat-like and light green in color, with all passaging or subculturing treatments. Interestingly, passage for 7 times through the insect host caused a yellow tint in the culture medium. Treated PSUM04 had similar colony morphology with flat and smooth mycelia, but with concentric dark green conidia, and it did not discolor the culture medium.

\section{Mycelial growth and spore production}

The mycelial growth rates of the treated and control M. guizhouense PSUM02 and PSUM04, observed at five day intervals, are shown in Table 1. Stock PSUM02 had a high mycelial growth rate by day 5 with $4.9 \pm 0.1 \mathrm{~mm} /$ day. At 10,15 and 20 days, the mycelial growth rates were similar across the fungal isolates and treatments. At the last observation day (20 days) the growth rate slightly decreased in all cases. As a general trend, the data suggest that successive subculturing weakens mycelia growth relative to the initial stock for PSUM02, while repeated passaging through an insect host slightly increases or restores it. In contrast, the treatment effects on growth of PSUM04 were negligible. These observations were checked from linear regression slopes, showing that successive subculturing significantly decreased the growth rate of PSUM02 ( $F=8.29$; df $=11$, 48; $P<0.01$ ), even though the difference was less than $10 \%$. The mycelial growth rate of PSUM04 was indeed not significantly affected by the treatments.

The spore production is graphically illustrated in Figure 1. M. guizhouense PSUM02 (Fig. 3A) had two clearly distinct groups, with its passage through an insect host 3 and 5 times matching stock/control in high spore production relative to the other treatments. The peak spore production in all cases occurred at 20 days after inoculation, and the highest value was $2.23 \pm 0.08 \times 10^{7} \mathrm{spore} / \mathrm{ml}$ for the stock culture. Treatment by subculturing 5 times on an artificial medium gave the lowest spore production on day 20 , with the $4.52 \pm 1.1 \times 10^{6}$ spore/ml being about five times lower than the control $(F=14.48 ; \mathrm{df}=5,24 ; P<0.05)$. 
The spore morphologies of the PSUM04 isolates were similar across the treatments (Fig. 3B); however, the levels of spore production differed between treatments. Unlike PSUM02, PSUM04 spore production was low until day 20. On day 20 the spore production of the stock/control and the fungi that had been passaged through insects were significantly higher $(F=$ 29.29; $\mathrm{df}=5,24 ; P<0.05)$ than those of the subcultured fungi. The peak spore production of the PSUM04 stock culture on day $20\left(6.91 \pm 2.27 \times 10^{7}\right.$ spore $\left./ \mathrm{ml}\right)$ was approximately 7.3 (resp. 6.3) folds relative to the spore production of PSUM04 subcultured 5 times $\left(9.49 \pm 0.22 \times 10^{6} \mathrm{spore} / \mathrm{ml}\right)$ (resp. 10 times, $1.09 \pm 0.11 \times 10^{7}$ spore/ml).

\section{Chitinase and protease production}

The enzymatic activities of treated and control M. guizhouense PSUM02 and PSUM04 are shown in Fig. 4. The PSUM02 had mostly no chitinase production, except when it was passed 5 or 7 times through an insect host, resulting in the enzymatic indexes $1.21 \pm 0.00$ and $1.54 \pm 0.10$, respectively. The PSUM04, regardless of treatment, had chitinase production with enzymatic indexes in the range $1.66-1.79(F=424.03$; $\mathrm{df}=11,36 ; P<0.01)$ (Fig. 4A).

The $M$. guizhouense PSUM04 showed significantly higher protease production than the PSUM02 $(F=386.49$; df $=11,36 ; P<0.01)$, consistently regardless of treatment, and the enzymatic indexes were in the range $1.79-2.00$ for PSUM04, and in the range $1.06-1.17$ for PSUM02 (Fig. 4B).

\section{Virulence of passaged M. guizhouense on C. cephalonica larvae}

The virulences across the varied culturing conditions of M. guizhouense PSUM02 and PSUM04 on $C$. cephalonica larvae are shown in Fig. 2 and Table 2. The treatments had statistically significant effects $(F=32.07$; $\mathrm{df}=12,117 ; P<0.05)$, and the Average Survival Time (AST) of $C$. cephalonica infected with $M$. guizhouense PSUM02 was significantly lower with serial passaging through an insect host 3, 5 or 7 times than with repeated subculturing on artificial media 10 times. With 7 passages of PSUM02 through an insect host, 100\% C. cephalonica larval mortality was reached within five days after infection. On the other hand, $99 \%$ larval mortality was only observed ten days after larval infection with PSUM02 serially subcultured on artificial media for 10 times.

The AST of C. cephalonica infected with PSUM04 serially passaged through an insect host 5 or 7 times showed similarly an increase in virulence. The PSUM04 was most pathogenic after 7 passages through an insect host, with $100 \%$ C. cephalonica larval mortality on day five, while after subculturing 10 times on artificial media, it gave $99 \%$ larval mortality only on day ten.

The results revealed that the virulence of $M$. guizhouense depends on the fungal isolate. The virulence of $M$. guizhouense PSUM02 decreased significantly with repeated subculturing on artificial media for 10 times, relative to the initial stock, but passaging through an insect host 3, 5 or 7 times had the opposite effect. The virulence of $M$. guizhouense PSUM04 was unaffected by repeated subculturing on artificial media 5 or 10 times, relative to the initial stock, and passaging through an insect host 5 or 7 times increased the virulence significantly.

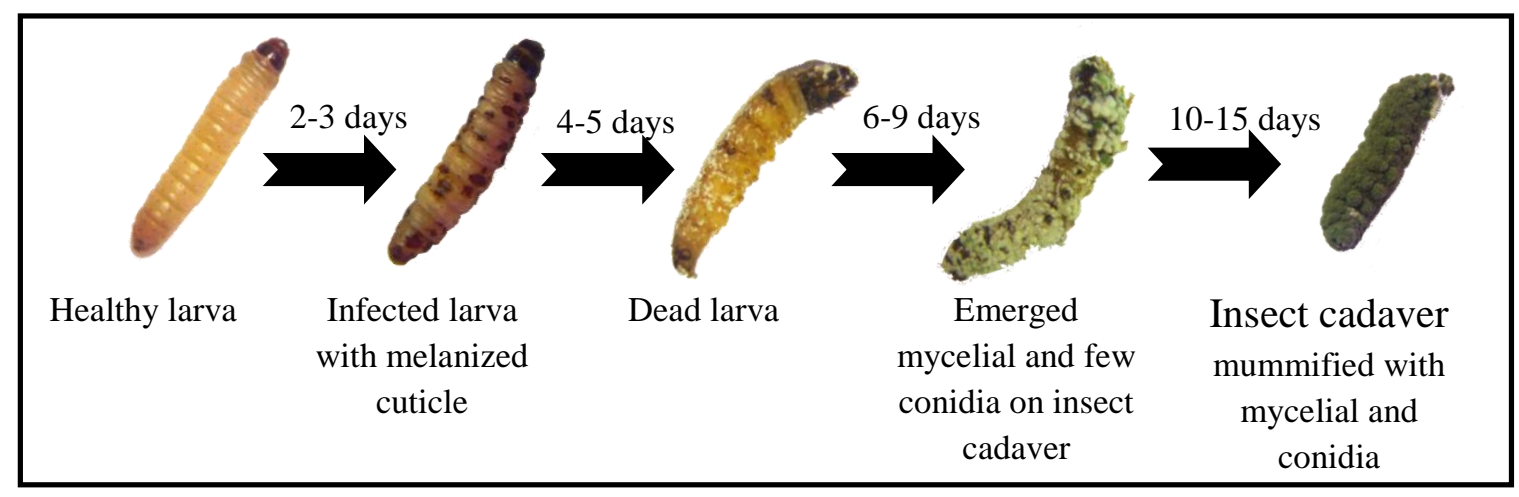

Fig. 2 - The infection process of Metarhizium guizhouense on rice moth larvae, Corcyra cephalonica. 

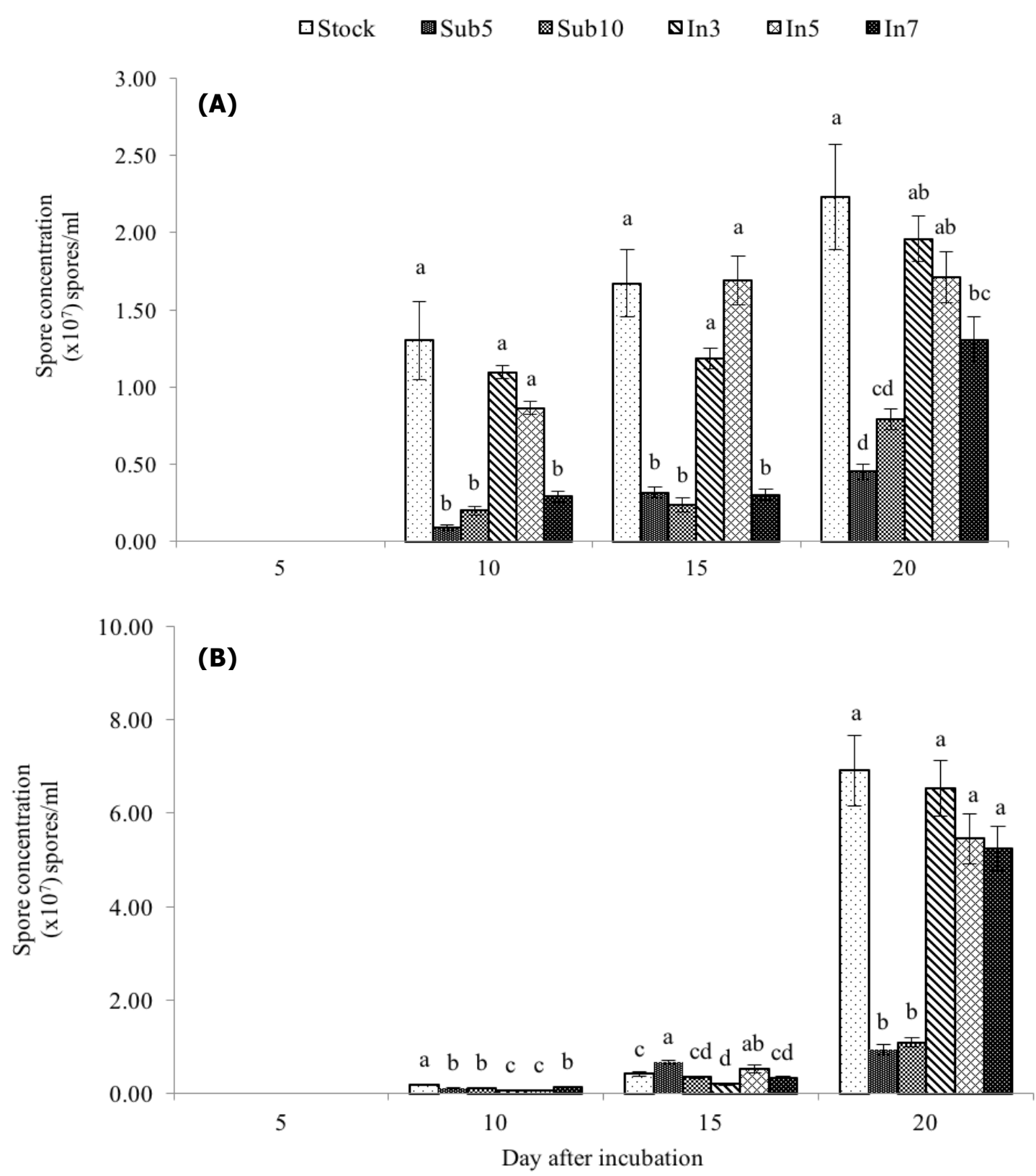

Fig. 3 - The spore production (mean \pm SE) of Metarhizium guizhouense PSUM02 (A) and PSUM04 (B), repeatedly passaged through $C$. cephalonica larvae or serially subcultured on artificial media, at 5, 10, 15 and 20 days after inoculation on SDAY media. Within each observation day different letters on the bars indicate statistically significant differences at the $P<0.01$ level, according to Tukey's HSD test. The treatment labels represent the following: Sub5 = subcultured on SDAY plates 5 times; Sub10 = subcultured on SDAY plates 10 times; In3 = infected through an insect 3 times; In5 = infected through an insect 5 times; and In7 = infected through an insect 7 times.

\section{Discussion}

Successive subculturing on artificial media and repeated passages through an insect host affected phenotypic and metabolic characteristics of M. guizhouense isolates PSUM02 and PSUM04, and effects on mycelial growth rate, sporulation, enzyme production and virulence were assessed. The various treatments had no significant effect on mycelial growth rate, while spore production was significantly affected. In particular, successive subculturing significantly reduced 


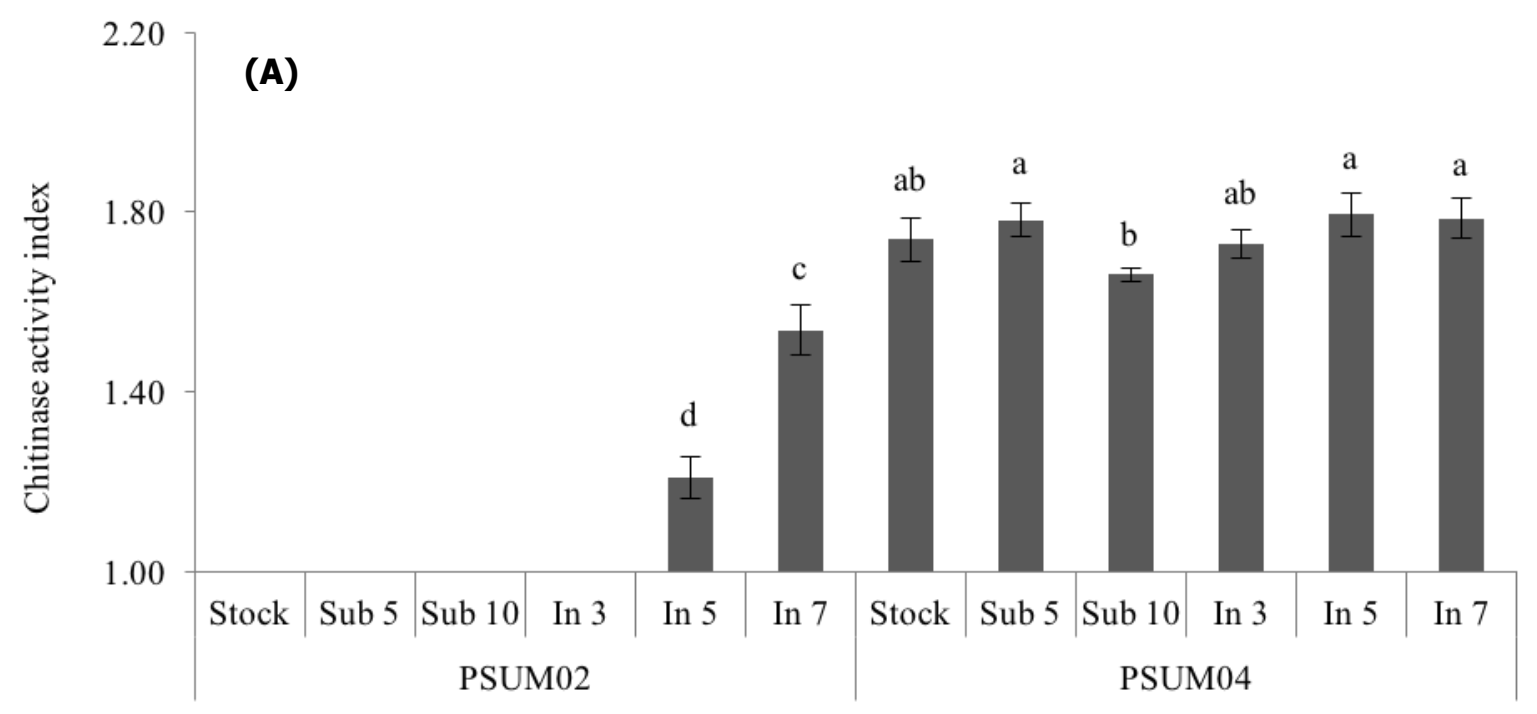

Treatments

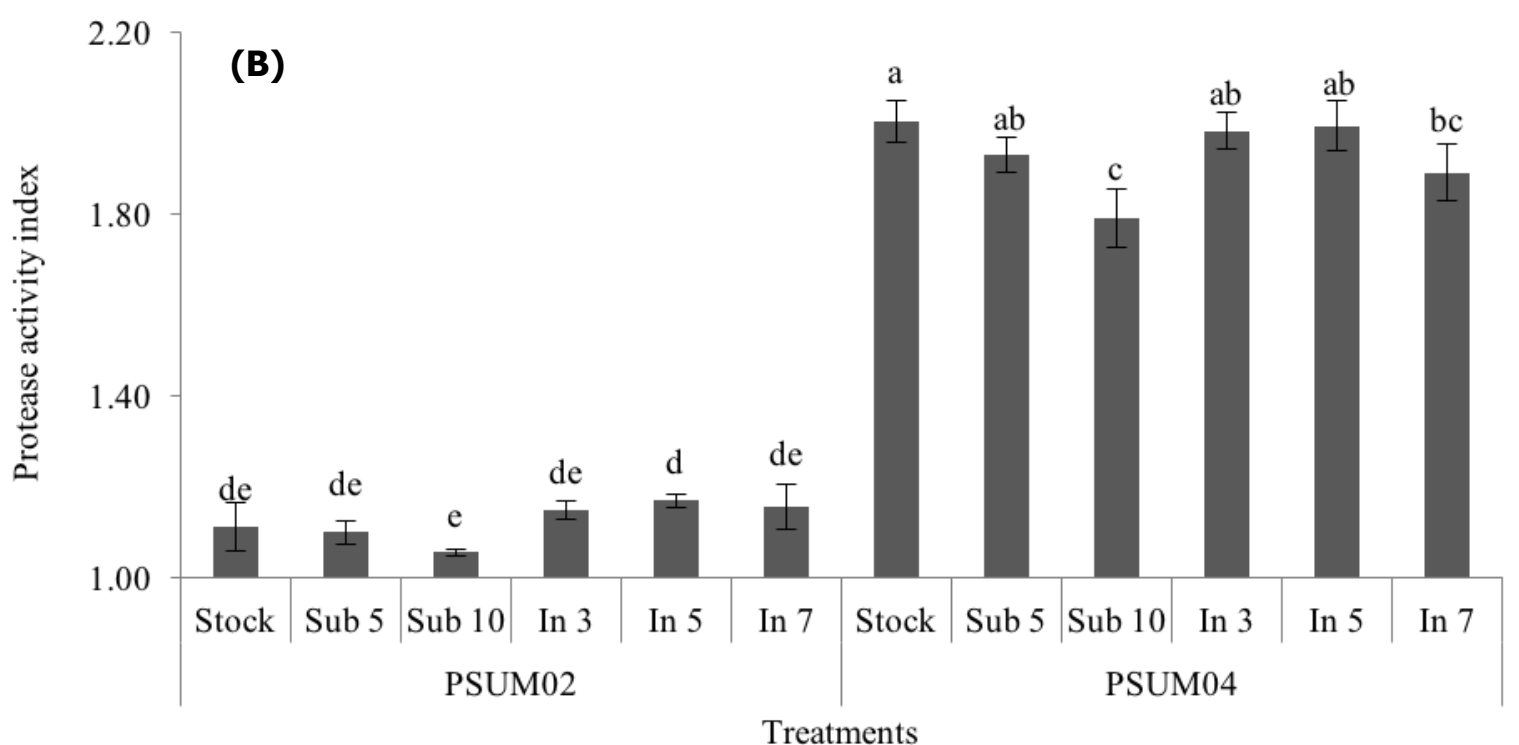

Fig. 4 - The chitinase (A) and protease (B) activity indexes of Metarhizium guizhouense PSUM02 and PSUM04, repeatedly passaged through $C$. cephalonica larvae or serially subcultured on artificial media. Enzyme activities were measured at 5 days post inoculation on the media. Each bar represents the mean $( \pm$ SD) of 4 replications. Different letters indicate statistically significant differences at the $P<0.01$ level, according to Tukey's HSD test. The treatment labels represent the following: Sub5 = subcultured on SDAY plates 5 times; Sub10 = subcultured on SDAY plates 10 times; In3 = infected through an insect 3 times; In $5=$ infected through an insect 5 times; and In7 = infected through an insect 7 times.

spore production, while passaging through insect hosts caused no deviation from the original fungal stock. Prior research confirms that subculturing entomopathogenic fungi, such as Beauveria bassiana, Isaria fumosorosea, M. anisopliae, M. brunneum and Paecilomyces fumosoroseus, on artificial media causes phenotypic alterations, including morphological changes in color, mycelial growth, and reduced sporulation, in agreement with our findings (Vandenberg \& Cantone 2004, Butt et al. 2006, Nahar et al. 2008, Hussain et al. 2010, Ansari \& Butt 2011, Safavi 2011, 2012). 
Table 2 Kaplan-Meier survival analysis of rice moth larvae (C. cephalonica) infected with Metarhizium guizhouense PSUM02 or PSUM04 repeatedly passaged through C. cephalonica larvae or serially subcultured on artificial media.

\begin{tabular}{|c|c|c|c|c|}
\hline \multirow{2}{*}{$\begin{array}{l}\text { Fungal } \\
\text { isolates }\end{array}$} & \multirow{2}{*}{ Treatments ${ }^{1}$} & \multirow{2}{*}{$\begin{array}{l}\text { Average survival time } \\
(\mathrm{AST})(\text { mean } \pm \mathrm{SE})^{2}\end{array}$} & \multicolumn{2}{|c|}{ 95\% Confidence interval } \\
\hline & & & Lower & Upper \\
\hline \multirow{6}{*}{ PSUM02 } & Stock & $3.96 \pm 0.20 \mathrm{de}$ & 3.58 & 4.34 \\
\hline & Sub 5 & $4.52 \pm 0.18 b c d$ & 4.17 & 4.87 \\
\hline & Sub 10 & $5.96 \pm 0.19 b$ & 5.59 & 6.33 \\
\hline & In 3 & $2.88 \pm 0.10 \mathrm{ef}$ & 2.68 & 3.08 \\
\hline & In 5 & $3.14 \pm 0.15 \mathrm{def}$ & 2.84 & 3.44 \\
\hline & In 7 & $2.29 \pm 0.12 \mathrm{f}$ & 2.06 & 2.52 \\
\hline \multirow{6}{*}{ PSUM04 } & Stock & $4.20 \pm 0.18 \mathrm{cde}$ & 3.85 & 4.55 \\
\hline & Sub 5 & $4.01 \pm 0.19 \mathrm{de}$ & 3.63 & 4.39 \\
\hline & Sub 10 & $5.67 \pm 0.16 b c$ & 5.35 & 5.99 \\
\hline & In 3 & $4.11 \pm 0.18 \mathrm{cde}$ & 3.76 & 4.46 \\
\hline & In 5 & $2.84 \pm 0.15 \mathrm{ef}$ & 2.55 & 3.13 \\
\hline & In 7 & $2.25 \pm 0.12 f$ & 2.02 & 2.48 \\
\hline \multicolumn{2}{|c|}{ Control (uninfected fungi) } & $10.00 \pm 0.00 \mathrm{a}$ & 10.00 & 10.00 \\
\hline
\end{tabular}

${ }^{1}$ Sub5 = subculture on SDAY plates 5 times; Sub10 = subculture on SDAY plates 10 times; In3 = infected through an insect 3 times; In $5=$ infected through an insect 5 times; and In $7=$ infected through an insect 7 times. Stock $=$ initial stock culture.

${ }^{2}$ Mean values followed by different letters within a column are statistically significantly different at the $P<0.01$ level, according to Tukey's HSD test. The AST was censored by limiting observations to 10 days.

As a general and consistent trend, passages through an insect host increased enzyme activity, while successive subculturing on an artificial medium decreased enzyme production relative to control (Shah et al. 2007). In our study, the fungal passage through an insect host or successive subculturing did not affect either chitinase or protease production of M. guizhouense PSUM02 or PSUM04. Other previous research has shown that the decline of enzyme production in a subculture does not correlate with virulence (Ansari \& Butt 2011). Extracellular enzymes produced by Metarhizium sp. are believed to play a key role in cuticle hydrolysis of insects, affecting virulence (Mustafa \& Kaur 2009).

Passaging through $C$. cephalonica larvae restored the virulence of $M$. guizhouense PSUM02 and increased it for PSUM04 relative to the original fungal stocks. Only the PSUM02 isolate that had been serially subcultured 10 times had decreased virulence, whereas PSUM04 isolate was not similarly affected. With 7 passages through an insect host, $M$. guizhouense PSUM02 gave 1.6 folds larval mortality relative to the original stock, and 9.0 fold larval mortality relative to the 10 times subcultured fungi, at three days post inoculation. The corresponding fold changes, also at three days post inoculation, were 2.1 and 14.2 for PSUM04. Adames et al. (2011) reported that 7 passages of $M$. anisopliae through an insect host increased its virulence to the tick Rhipicephalus microplus 1.3 folds relative to the untreated control, and four passages had a 1.1 folds effect, at the constant spore concentration of $1 \times 10^{8}$ spore/ml. Frazzon et al. (2000) also report that the number of entomopathogenic fungi passages through a suitable host correlates with increased virulence and reduced attenuation.

The enzyme activities of chitinases and proteases are not the only determinants of the virulence of $M$. guizhouense; toxins (destruxins) are also important. The number of passages through an insect host could induce more destruxin production than repeated subculturing on culture media. It is possible that the observed mortality of $C$. cephalonica was due to an increase in destruxin. Seven passages of $M$. guizhouense through an insect host increased its virulence toward C. cephalonica larvae by more than $30 \%$ in the larval mortality within 24 hours. It has been 
reported that the most virulent strains of Metarhizium sp. produce large quantities of destruxins (Amiri-Besheli et al. 2000), and this toxin is known to cause muscle paralysis resulting in the death of the insect (Pedras et al. 2002). Death occurs shortly after inoculation and often without any sign of the fungus in the haemocoel, suggesting that the toxins are secreted during very early stages of infection (Butt et al. 1994) and destruxins could influence the rate at which the insect host dies (Amiri-Besheli et al. 2000). On the other hand, the destruxins production in vitro of Metharhizium spp. need not correlate with its virulence to an insect (Golo et al. 2014). Some isolates of Metharhizium spp. with destruxins below detection limit were most virulent to the insect, and presence or absence of destruxins in vitro had little or no correlation with the mortality or with the speed of insect kill (Golo et al. 2014).

Moreover, repeated subculturing of the fungus on culture media decreased virulence, in agreement to the work of Shah et al. (2007), Hussain et al. (2010), and Safavi (2011, 2012). The mortality of $C$. cephalonica larvae infected with 10 times subcultured M. guizhouense was less than $10 \%$ at three days post inoculation. Shah et al. (2007) reported that repeated subculturing caused rapid changes in some virulence properties of $M$. anisopliae, such as conidial adhesion and the protease spore-bound Pr1. These properties were directly correlated with fungal virulence. It has been suggested that cuticle-degrading enzymes such as chitinase, CDA, chitosanase and protease (Nahar et al. 2008) or destruxins (Butt et al. 2006) could be used as markers to monitor the virulence of entomopathogenic fungi during mass production.

Overall, these results suggest that 3 to 5 passages through an insect host, for both $M$. guizhouense PSUM02 and PSUM04 entomopathogenic fungi, can help to restore or increase fungal virulence, which is important biological control agent in insect pest infection.

\section{Acknowledgements}

We would like to thank the Thailand Research Fund (Grant MRG5580113) for their financial support and the Department of Pest Management, Faculty of Natural Resources, Prince of Songkla University and the Natural Biological Control Research Center, Southern Region for allowing us to use their facilities and equipment. We would like to thank the Research and Development Office, Prince of Songkla University and Assoc. Prof. Dr. Seppo Karrila, Faculty of Science and Industrial Technology, Prince of Songkla University, Surat Thani Campus, for editing our manuscript.

\section{References}

Adames M, Fernández-Ruvalcaba M, Pená-Chora G, Hernández-Velázquez VM. 2011 - Effects of passages through a suitable host of the fungus, Metarhizium anisopliae, on the virulence of acaricide-susceptible and resistant strains of the tick, Rhipicephalus microplus. Journal of Insect Science 11, 1-13.

Amiri-Besheli B, Khambay B, Cameron S, Deadman ML, Butt TM. 2000 - Inter- and intra-specific variation in deatruxin production by insect pathogenic Metarhizium spp., and its significance to pathogenesis. Mycological Research 104, 447-452.

Ansari MA, Butt TM. 2011 - Effects of successive subculturing on stability, virulence, conidial yield, germination and shelf-life of entomopathogenic fungi. Journal of Applied Microbiology 110, 1460-1469.

Balachander M, Remadevi OK, Sasidharan TO, Bai NS. 2012 - Virulence and mycotoxic effects of Metarhizium anisopliae on Mahogany shoot borer, Hypsipyla robusta (Lepidoptera: Pyralidae). Journal of Forest Research 23, 651-659.

Bidochka MJ, Kamp AM, Lavender TM, de Koning J, de Grooss JNA. 2001 - Habitat association of two genetic groups of the insect-pathogenic fungus Metarhizium anisopliae: uncovering cryptic species?. Applied and Environmental Microbiology 67, 1335-1342. 
Bidochka MJ, Small CLN, Spironello M. 2005 - Recombination within sympatric cryptic species of the insect-pathogenic fungus Metarhizium anisopliae. Environmental Microbiology 7 , 1361-1368.

Bischoff JF, Rehner SA, Humber RA. 2009 - A multilocus phylogeny of the Metarhizium anisopliae lineage. Mycologia 101, 512-530.

Boucias DG, Pendland JC. 1998 - Principle of Insect Pathology, Kluwer Academic Publishers, Boston.

Brownbridge M, Costa S, Jaronski ST. 2001 - Effects of in vitro passage of Beauveria brassiana on virulence to Bemisia argentifolii. Journal of Invertebrate Pathology 77, 280-283.

Butt TM, Ibrahim L, Ball BV, Clark SJ. 1994 - Pathogenicity of the entomopathogenous fungus Metarhizium anisopliae and Beauveria bassiana against crucifer pests and the honey bee. Biocontrol Science and Technology 4, 207-214.

Butt TM, Wang C, Shah FA, Hall R. 2006 - Degeneration of entomogenous fungi, in: Eilenberg, J., Hokkanen, H.M.T. (Eds.), An Ecological and Societal Approach to Biological Control. Springer, Netherlands, pp. 213-226.

Geng B, Zhang R. 2004 - Pathogenicity of Metarhizium anisopliae var. acridu to the developmental stages of brown planthopper Nilaparvata lugens Stal and Sogatella furcifera (Horvath). Entomologia Sinica 11, 89-97.

Golo PS, Gardner DR, Grilley MM, Takemoto JY, Krasnoff SB, Pires MS, Fernandes ÉKK, Bittencourt VREP, Robert DW. 2014 - Production of destruxins from Metarhizium spp. fungi in endophytically colonized cowpea plants. PLoS One, 9, e104946.

Frazzon AP, Vaz Junior I, Masuda A, Schrank A, Henning M. 2000 - In vitro assessment of Metarhizium anisopliae isolates to control the cattle tick Boophilus microplus. Veterinary Parasitology 94, 117-125.

Hussain A, Tian MY, He YR, Ruan L, Ahmed S. 2010 - In vitro and in vivo culturing impacts on the virulence characteristics of serially passed entomopathogenic fungi. Journal of Food Agriculture and Environment 8, 481-487.

Jagadish PS, Nirmala P, Rashmi MA, Hedge JN, Nangia N. 2009 - Biology of rice moth, Corcyra cephalonica Stainton on foxtail millet. Karnataka Journal of Agricultural Science 22, 674675.

Jonason NB, Boetel MA, Eide JD, Campbell LG, Rao MB. 2005 - Virulence of Metarhizium anisopliae (Deuteromycotina: Hyphomycetes) to sugarbeet root maggot (Diptera: Ulidiidae) larvae. Journal of Sugar Beet Research 42, 103-117.

Khashaveh A, Safaralizadeh MH, Ghosta Y. 2011 - Pathogenicity of Iranian isolates of Metarhizium anisopliae (Metschinkoff) (Ascomycota: Hypocreales) against Trogoderma granarium Everts (Coleoptera: Dermestidae). Biharean Biologist 5, 51-55.

Loc NT, Chi VTB. 2007 - Biocontrol potential of Metarhizium anisopliae and Beauveria bassiana against diamondback moth, Plutella xylostella. Omonrice 15, 86-93.

Lui H, Bauer LS. 2006 - Susceptibility of Agrilus planipennis (Coleoptera: Buprestidae) to Beauveria bassiana and Metarhizium anisopliae. Journal of Economic Entomology 99, 1096-1103.

Mustafa U, Kaur G. 2009 - Extracellular enzyme production in Metarhizium anisopliae isolates. Folia Microbiologica 54, 499-504.

Nahar PB, Kulkarani SA, Kulye MS, Chavan SB, Kulkarani G, Rajendran A, Yadav PD, Shouche Y, Deshpande MV. 2008 - Effect of repeated in vitro subculturing on the virulence of Metarhizium anisopliae against Helicoverpa armigera (Lepidoptera: Noctuidae). Biocontrol Science and Technology 18, 337-355.

Parra JRP, Zucchi RA, Silveira NS, Haddad ML. 1991 - Biology and thermal requirements of Trichogramma galloi and T. distinctum on two alternative hosts. Colloques-INRA 56, 8184.

Pedras MCSC, Zaharia LI, Ward DE. 2002 - The destruxins: synthesis, biosynthesis, biotransformation and biological activity. Phytochemistry 59, 579-596. 
Petlamul W, Prasertsan P. 2012 - Evaluation of strain of Metarhizium anisopliae and Beauveria bassiana against Spodoptera litura on the basis of their virulence, germination rate, conidia production, radial growth and enzyme activity. Mycobiologia 40, 111-116.

Safavi SA. 2011 - Successive subculturing alters spore-bound Pr1 activity, germination and virulence of the entomopathogenic fungus, Beauveria bassiana. Biocontrol Science and Technology 21, 883-890.

Safavi SA. 2012 - Attenuation of the entomopathogenic fungus Beauveria bassiana following serial in vitro transfers. Biologia 67, 1062-1068.

Shah FA, Allen A, Wright CJ, Butt TM. 2007 - Repeated in vitro subculturing alters spore surface properties and virulence of Metarhizium anisopliae. FEMS Microbiology Letters 276, 6066.

Shah FA, Wang CS, Butt TM. 2005 - Nutrition influences growth and virulence of the insect pathogenic fungus Metarhizium anisopliae. FEMS Microbiology Letters 251, 259-266.

Song TT, Feng MG. 2011 - In vivo passages of heterologous Beauveria brassiana isolates improve conidial surface properties and pathogenicity to Nilaparvata lugens (Homoptera: Delphacidae). Journal of Invertebrate Pathology 106, 211-216.

SPSS. 2001 - SPSS for Windows 11. SPSS Inc., Chicago, IL, USA. URL http://www.spss.com.

St Leger RJ, Joshi L, Roberts DW. 1997 - Adaptation of proteases and carbohydrates of saprophytic, phytopathogenic and entomopathogenic fungi to the requirements of their ecological niches. Microbiology 143, 1983-1992.

Tavassoli M, Allymehr M, Pourseyed SH, Ownag A, Bernousi I, Mardani K, Ghorbanzadegan M, Shokrpoor S.2011 - Field bioassay of Metarhizium anisopliae strains to control the poultry red mite Dermanyssus gallinae. Veterinary Parasitology 178, 374-378.

Thaochan N, Ngampongsai A. 2015 - Effects of autodisseminated Metarhizium guizhouense PSUM02 on mating propensity and mating competitiveness of Bactrocera cucurbitae (Diptera: Tephritidae). Biocontrol Science and Technology 25, 629-644.

Vandenberg JD, Cantone FA. 2004 - Effect of serial transfer of three strains of Paecilomyces fumosoroseus on growth in vitro, virulence and host specificity. Journal of Invertebrate Pathology 85, 40-45.

Wekesa VW, Maniania NK, Knapp M, Boga HI. 2005 - Pathogenicity of Beauveria bassiana and Metarhizium anisopliae to the tobacco spider mite Tetranychus evansi. Experimental and Applied Acarology 36, 41-50.

Yousef M, Lozano-Tovar MD, Garrido-Jurado I, Quesada-Moraga E. 2013 - Biocontrol of Bactrocera oleae (Diptera: Tephritidae) with Metarhizium brunneum and its extracts. Journal of Economic Entomology 106, 1118-1125. 